\title{
The significance of aspartate on $\mathrm{NAD}(\mathrm{H})$ biosynthesis and $A B E$ fermentation in Clostridium acetobutylicum ATCC 824
}

\author{
Zhengping Liao ${ }^{1}$, Xitong Yang ${ }^{1}$, Hongxin Fu ${ }^{1 *}$ and Jufang Wang ${ }^{1,2^{*}}$ (D)
}

\begin{abstract}
The co-factor NADH plays an important role in butanol biosynthesis. In this study, we found that aspartate could effectively improve the butanol production of Clostridium acetobutylicum ATCC 824. Further study showed that aspartate could be used as the precursor of NADH de novo synthesis in C. acetobutylicum ATCC 824. When $2 \mathrm{~g} / \mathrm{L}$ aspartate was added, the transcription levels of essential genes ( adA, nadB and nadC) for NADH de novo synthesis were significantly higher than that of without aspartate addition. The levels of intracellular NAD ${ }^{+}, N A D H$, total $N A D(H)$ and the ratio of $\mathrm{NADH} / \mathrm{NAD}^{+}$were also significantly increased, which were $63.9 \pm 8.0 \%, 85.0 \pm \%, 77.7 \pm 8.0 \%$ and $12.7 \pm 2.9 \%$ higher than those of without aspartate addition, respectively. Furthermore, the butanol production was improved by overexpressing the NADH de novo synthesis genes, and the fermentation performance could be further enhanced by strengthening the VB1 biosynthesis and NADH de novo synthesis pathway simultaneously. As a result, the butanol titer of the engineered strain 824(thiCGE-nadC) reached $13.96 \pm 0.11 \mathrm{~g} / \mathrm{L}, 7.2 \pm 0.4 \%, 18.1 \pm 0.1 \%, 34.1 \pm 0.1 \%$ higher than that of 824 (thiCGE), 824(nadC) and the wild type strain, respectively. This study has a reference value for the $\mathrm{NADH}$ related researches of other microbes, and the engineering strategy used in this study provides a new idea for construction of efficient fuel-producing strains.
\end{abstract}

Keywords: Clostridium acetobutylicum, Butanol, Aspartate, NADH

\section{Introduction}

With the exhaustion of fossil energy and the deterioration of global environmental problems, the biofuels have been attracted more and more attention. As a biofuel, the physical and chemical properties of butanol are superior to ethanol and similar to gasoline, thus it could be used as a fuel additive or as a replacement of gasoline (Lee et al. 2008). However, the market competitiveness of biobutanol is weak due to the low titer, yield and proportion of butanol in traditional ABE fermentation. Therefore, improving the butanol production is of great significance to the butanol fermentation industry.

In organisms, most of the physiological and biochemical processes are achieved through redox reactions.

\footnotetext{
*Correspondence: hongxinfu@scut.edu.cn; jufwang@scut.edu.cn

${ }^{1}$ School of Biology and Biological Engineering, South China University

of Technology, Guangzhou 510006, China

Full list of author information is available at the end of the article
}

Cofactor NADH is essential in these redox reactions. For example, NADH was involved in 43 reactions in Escherichia coli and 65 reactions in Saccharomyces cerevisiae (Nielsen 2003). Therefore, redox balance is of great significance for maintaining normal cell growth and physiological metabolism, and NADH plays an important role in maintaining the redox balance (Qi et al. 2018). In addition, NADH also could affect the products formation and metabolic flux redistribution. For example, the succinic acid production by $E$. coli and the ethanol production by Clostridium thermophilus can be improved by increasing the supply of NADH (Balzer et al. 2013; Biswas et al. 2015). It was reported that NADH is one of the key factors in the transformation from acidogenic phase to solventogenic phase of ABE (acetone-butanol-ethanol) fermentation by $C$. acetobutylicum, and the redox balance could determine the metabolic flux redistribution (Peguin et al. 1994; Wietzke and Bahl 2012). In C. 
acetobutylicum, the biosynthesis of butanol is strongly dependent on the availability of cofactor NADH. In general, the synthesis of butanol is an NADH consuming process: $1 \mathrm{~mol}$ of glucose can produce $2 \mathrm{~mol}$ of NADH through glycolysis, while $4 \mathrm{~mol} \mathrm{NADH}$ are required for $1 \mathrm{~mol}$ of butanol synthesis. The NADH produced in glycolysis cannot meet the demand of butanol synthesis, thus insufficient NADH supply have limited the synthesis of butanol. Therefore, it is an effective way to improve butanol production by increasing the NADH supply in $C$. acetobutylicum. In recent years, a lot of work in improving the concentration of NADH for biofuels production have been reported. Formic acid dehydrogenase (FDH) can oxidize formic acid into carbon dioxide and generate NADH. Therefore, the NADH levels and butanol production were significantly improved by introducing FDH into E. coli (Lim et al. 2013; Shen et al. 2011). However, the NADH supply was not significantly affected after heterogenously expressed FDH from Candida boidinii in $C$. acetobutylicum (Wang et al. 2011). Hydrogenase has a great influence on NADH formation because it can compete with ferredoxin for electrons to produce hydrogen. Therefore, inhibiting the activity of hydrogenase could be used as an effective strategy to improve NADH supply. For example, Biswas' research showed that knocking out the hydrogenase gene of Clostridium thermocellum resulted in an increasement of the ethanol titer and yield (Biswas et al. 2015). However, it is difficult to knockout hydrogenase gene in butanol producing Clostridium, or its effect on NADH formation and product distribution was very limited, even the hydrogenase gene knockout was achieved (Cooksley et al. 2012; Jang et al. 2014; Liu et al. 2015). In addition, the NADH and ATP levels of Clostridium beijerinckii NCIMB 8052 was significantly increased by insertional inactivation of the NADHquinone oxidoreductase gene Cbei_4110, resulting in a 21.8\% improved in butanol titer (Liu et al. 2016). NAD(P) $\mathrm{H}$ availability was also improved by overexpressing FdNR (ferredoxin $\mathrm{NAD}(\mathrm{P})^{+}$oxidoreductase) in the C. acetobutylicum buk $^{-}$strain, which resulted in an improvement in the production of butanol and the ratio of butanol/acetate (Qi et al. 2018).

However, there were few researches focused on increasing the NADH levels of solventogenic clostridia by enhancing the de novo synthesis pathways of $\mathrm{NADH}$. In organism, NADH was synthesized by means of de novo pathway and salvage pathway (Fig. 1). There are two de novo synthesis pathways of NADH, namely Asp pathway and Kynurenine pathway. Asp pathway used aspartate as the precursor, and Kynurenine pathway used tryptophan as the precursor (Akira et al. 2006) (Fig. 1). The way of NADH de novo synthesis differs in different microorganisms. For example, in most eukaryotes (e.g., Saccharomyces cerevisiae, Streptomyces antigens), NADH is synthesized with tryptophan as the precursor (Kurnasov et al. 2003); while in most prokaryotes (such as E. coli), NADH is synthesized with aspartate as the precursor (Akira et al. 2006). Based on

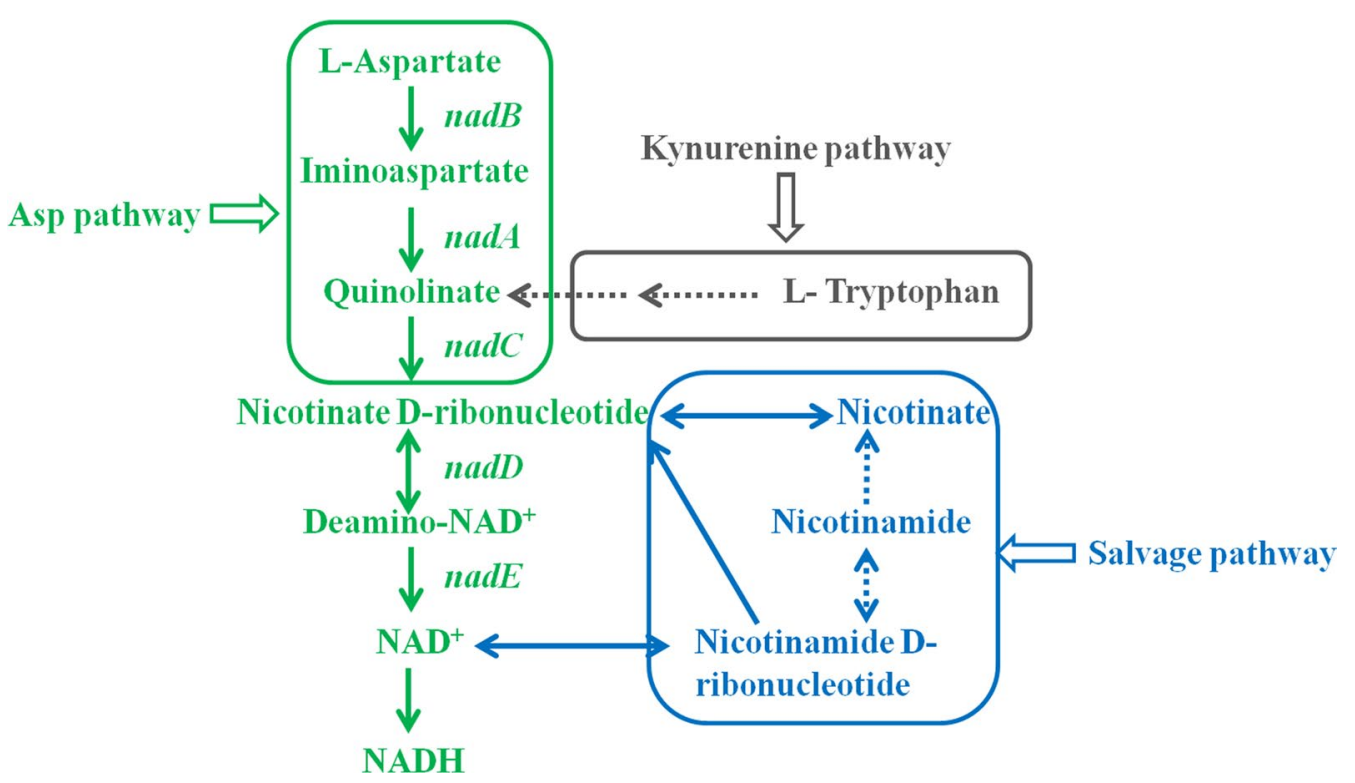

Fig. 1 NADH biosynthetic pathway of Clostridium acetobutylicum ATCC 824. The gray section means that Kynurenine pathway does not exist in Clostridium acetobutylicum ATCC 824. nadB, L-aspartate oxidase gene; nadA, quinolinate synthetase gene; nadC, nicotinate-nucleotide pyrophosphorylase gene; nadD, nicotinic acid mononucleotide adenylyltransferase; nadE, NAD synthetase 
the genomic sequencing data (Nölling et al. 2001), there are no Kynurenine pathway genes in genome of $C$. acetobutylicum ATCC 824. Therefore, we speculated that the de novo synthesis of $\mathrm{NADH}$ in $C$. acetobutylicum ATCC 824 is by Asp pathway, rather than by Kynurenine pathway. As shown in Fig. 1, aspartate is catalyzed by aspartate oxidase (NadB), quinolinate synthetase (NadA) and nicotinate-nucleotide pyrophosphorylase (NadC) to form nicotinate D-ribonucleotide (NaMN). Subsequently, NaMN is catalyzed by nicotinic acid mononucleotide adenylyltransferase (NadD) and NAD synthase (NadE) to form $\mathrm{NAD}^{+}$, these two enzymes are common to both the de novo pathway and salvage pathway. Therefore, nadA (cac1025), nadB (cac1024) and nadC (cac1023) are essential genes for de novo synthesis of NADH in C. acetobutylicum ATCC 824.

In this study, the effect of aspartate on ABE fermentation and $\mathrm{NAD}(\mathrm{H})$ biosynthesis of $C$. acetobutylicum ATCC 824 was investigated firstly. Then, the essential genes ( $\operatorname{nad} A$, nadB or $n a d C$ ) for NADH de novo synthesis were overexpressed to improve the butanol production. Moreover, to further increase the butanol production, both the biosynthesis pathway of NADH and VB1 (thiC, thiG and thiE) were enhanced in C. acetobutylicum ATCC 824. The results showed that both aspartate addition and NADH de novo synthesis genes overexpression could promote butanol production and NAD $(\mathrm{H})$ biosynthesis. Moreover, co-expression of nadC, thiC, thiG and thiE could further improve the butanol production of C. acetobutylicum ATCC 824 . This work revealed that enhancing Asp pathway could improve the butanol production of $C$. acetobutylicum ATCC 824 by promoting $\mathrm{NAD}(\mathrm{H})$ biosynthesis, providing a new and effective engineering target for solventogenic Clostridia.

\section{Materials and methods}

Strains, plasmids and primers

The strains and plasmids used in this study are shown in Table 1 . The plasmid pMTL82151 is an E. coli-Clostridium shuttle plasmid, and the plasmid pAN2 contains a methyltransferase gene $\Phi 3 t$ I. C. acetobutylicum ATCC 824 , which used as a wild type strain, was provided by Professor Shang-Tian Yang (The Ohio State University). All of $E$. coli strains were purchased from TIANGEN (Beijing, China). E. coli DH5 $\alpha$ was used for plasmids amplification and $E$. coli TOP10 (containing pAN2) was used for recombinant plasmids methylation in vivo. All of the primers used in this study (Table 2) were synthesized by TIANYI HUIYUAN (Guangzhou, China).

\section{Culture conditions}

Escherichia coli strains were cultured aerobically with Luria-Bertani (LB) medium supplemented with $20 \mathrm{mg} / \mathrm{L}$ tetracycline or/and $25 \mu \mathrm{g} / \mathrm{mL}$ chloramphenicol if needed, and $C$. acetobutylicum strains were cultured anaerobically with reinforced clostridial medium (RCM) (Ventura et al. 2013) supplemented with $30 \mathrm{mg} / \mathrm{L}$ thiamphenicol if needed. P2 medium (containing $80 \mathrm{~g} / \mathrm{L}$ glucose) (Liao et al. 2017) was used for the batch fermentation of C. acetobutylicum. Both of E. coli and C. acetobutylicum were incubate at $37^{\circ} \mathrm{C}$.

\section{Recombinant plasmid construction and transformation}

The genome DNA of C. acetobutylicum ATCC 824 was extracted using AxyPrep ${ }^{\mathrm{TM}}$ Genomic DNA Kit (Corning, Wujiang, China). The NADH de novo synthesis genes nadA, andB, nadC or nadC' was isolated from the genome DNA of C. acetobutylicum ATCC 824 by PCR, the primers used for gene amplification are shown in Table 2. Subsequently, the PCR products were purified

Table 1 Strains and plasmids used in this study

\begin{tabular}{|c|c|c|}
\hline Strains and plasmids & Characteristics & Sources and references \\
\hline \multicolumn{3}{|l|}{ Strains } \\
\hline C. acetobutylicum ATCC 824 & Wild type & ATCC \\
\hline E. coli DH5a & DeoR, recA1, endA1, hsdR17 $\left(\mathrm{rk}^{-}, \mathrm{mk}^{+}\right)$ & TIANGEN \\
\hline E. coli Top10 & hsd $R, \operatorname{mcr} A$, rec $A 1$, end $A 1$ & TIANGEN \\
\hline \multicolumn{3}{|l|}{ Plasmids } \\
\hline pAN2 & Ф3t I, p15A ori, Tet ${ }^{R}$ & Heap et al. (2007) \\
\hline pMTL82151 & ColE1 ori; $\mathrm{Cm}^{\mathrm{R}}$; pBP1 ori; TarJ & Heap et al. (2009) \\
\hline pMTL-Pthl & From pMTL82151; P-thl & Liao et al. (2018) \\
\hline pMTL-Pthl thiCGE & From pMTL82151; P-thl::thic + thiG + thiE & Liao et al. (2018) \\
\hline pMTL-Pthl-nadA & From pMTL82151; P-thl::nadA & This work \\
\hline pMTL-Pthl-nadB & From pMTL82151; P-th/::nadB & This work \\
\hline pMTL-Pthl-nadC & From pMTL82151; P-thl::nadC & This work \\
\hline pMTL-Pthl-thiCGE-nadC & From pMTL82151; P-thl::thiC + thiG + thiE + nadC & This work \\
\hline
\end{tabular}


Table 2 Primers used in this study

\begin{tabular}{|c|c|c|}
\hline Primers & Sequence $\left(5^{\prime}-3^{\prime}\right)$ & Sources and references \\
\hline \multicolumn{3}{|c|}{ Primers for gene amplification } \\
\hline $\operatorname{nadA}-\mathrm{F}$ & AGGAGGTTAGTTAGA GGATCC TCACATACACCCCTTATTTCCAAC & This work \\
\hline nadA-R & ACGACGGCCAGTGCC AAGCTTTCACCTTCCAAGTATTAACATATTT & This work \\
\hline$n a d B-F$ & AGGAGGTTAGTTAGA GGATCC ATGAATATTCAAACTGACGTATTAAT & This work \\
\hline$n a d B-R$ & ACGACGGCCAGTGCC AAGCTTTCAAATGTTGACCAATTCATTTTTTC & This work \\
\hline $\operatorname{nadC}-\mathrm{F}$ & AGGAGGTTAGTTAGA GGATCC ATGAATTGGTCAACATTTGATGAT & This work \\
\hline nadC-R & ACGACGGCCAGTGCC AAGCTTTTATTTTTCATTTCTTAAGTTTTTCATGC & This work \\
\hline $\mathrm{NadC}-\mathrm{F}$ & ACATCCCCCTTTCGC CAGCTG GAATCCATTTTGGGGGAAAAG & This work \\
\hline $\mathrm{NadC}-\mathrm{R}$ & CAGGCTTCTTATTTTTTAT GCTAGC TTATTTTTCATTTCTTAAGTTTTTTCATGC & This work \\
\hline PMTL-F & TGAAGTACATCACCGACGAGCAAG & This work \\
\hline PMTL-R & TGCTGCAAGGCGATTAAGTTGGGT & This work \\
\hline PMTL-R' & CCTGTTGAACCATTAGCTAAGGA & This work \\
\hline \multicolumn{3}{|c|}{ Primers for RT-PCR } \\
\hline $\operatorname{nad}(\mathrm{RT})-\mathrm{F}$ & CAAAGACCTGAGGTGCAGGAA & This work \\
\hline $\operatorname{nadA}(\mathrm{RT})-\mathrm{R}$ & GCCATTGGACAGCCAGCTT & This work \\
\hline $\operatorname{nadB}(\mathrm{RT})-\mathrm{F}$ & CTTCAGGCGGTATAGGTGG & This work \\
\hline $\operatorname{nadB}(\mathrm{RT})-\mathrm{R}$ & TCGCGAACGTCTATGTTATG & This work \\
\hline $\operatorname{nadC}(\mathrm{RT})-\mathrm{F}$ & AAACGCTTAGAGGGCACAGG & This work \\
\hline $\operatorname{nadC}(\mathrm{RT})-\mathrm{R}$ & TGCCGTCAGAAAGACCAAATC & This work \\
\hline CAC2679-F & GACATTACTTCAAACGAACCTG & Liao et al. (2018) \\
\hline CAC2679-R & CCCTTAGCCCATTTATTCCT & Liao et al. (2018) \\
\hline
\end{tabular}

and $n a d A$, andB or nadC was inserted into the shuttle vector pMTL-Pthl, which was digested with restriction enzymes BamHI and HindIII (Thermo Scientific, Shanghai, China), and $n a d C^{\prime}$ was inserted into the recombinant plasmid pMTL-Pthl thiCGE, which was digested with restriction enzymes PvuII and NheI (Thermo Scientific, Shanghai, China), then the recombinant plasmids pMTLPthl nadA, pMTL-Pthl nadB, pMTL-Pthl nadC and pMTL-Pthl thiCGE-nadC were obtained. The method of ligation was according to the instruction of ClonExpress II One Step Cloning Kit (Vazyme, Nanjing, China). Before transferring into C. acetobutylicum ATCC 824, the recombinant plasmids must be methylated by transforming into $E$. coli TOP10 (containing plasmid pAN2). Then, the methylated plasmids could be transferred into C. acetobutylicum ATCC 824, the transformation method was described previously (Liao et al. 2017). The transformants of 824(nadA), 824(nadB) and 824(nadC) were identified by PCR with primers pMTL-F/pMTL-R and transformants of 824(thiCGE-nadC) was identified with primers pMTL-F/pMTL-R' (Table 2).

\section{RNA extraction and RT-PCR analysis}

Clostridium acetobutylicum ATCC 824 was inoculated in liquid RCM for 12-24 h, then transferred to fresh RCM (inoculum size was $10 \% \mathrm{v} / \mathrm{v}$ ) and cultured to logarithmic phase. The seed was inoculated in P2 medium without or with $2.0 \mathrm{~g} / \mathrm{L}$ aspartate. The samples were taken at 12, 24, and $36 \mathrm{~h}$ and used for total RNA extraction. The method of total RNA extraction was according to the instruction of RNAprep pure Cell/Bacteria Kit (Tiangen Biotech, Beijing, China). cDNA was synthesized using total RNA as the template, and the detailed operation procedures was according to the instruction of PrimeScript ${ }^{\mathrm{TM}} \mathrm{RT}$ reagent Kit with gDNA Eraser (Takara, Dalian, China). Real time PCR (RT-PCR) analysis used cDNA (diluted 10 times) as the template, and detailed operation procedures was according to the instruction of SYBR Premix Ex Taq II $(2 \times)$ (Tli RNaseH Plus), Bulk (Takara, Dalian, China). The primers for target genes and housekeeping gene (CAC2679) (Tomas et al. 2003; Tseng et al. 2001) are listed in Table 2.

\section{Effect of exogenous aspartate addition on intracellular NAD(H) level of C. acetobutylicum ATCC 824}

Clostridium acetobutylicum ATCC 824 was inoculated in liquid RCM for $12-24 \mathrm{~h}$, then transferred to fresh RCM (inoculum size was $10 \% \mathrm{v} / \mathrm{v}$ ) and cultured to the logarithmic phase. The seed was inoculated in P2 medium without or with $2.0 \mathrm{~g} / \mathrm{L}$ aspartate. The samples were taken at $24 \mathrm{~h}$ and used for extraction of $\mathrm{NAD}^{+}$and $\mathrm{NADH}$. The bacteria were collected by centrifugation $\left(4{ }^{\circ} \mathrm{C}, 12,000 \mathrm{rpm}\right.$ for $5 \mathrm{~min}$ ) from $1 \mathrm{~mL}$ of the samples, then resuspend the bacteria with $0.3 \mathrm{~mL}$ of $0.2 \mathrm{M} \mathrm{NaOH}$ 
(for $\mathrm{NADH}$ extraction) or $\mathrm{HCl}$ (for $\mathrm{NAD}^{+}$extraction). Subsequently, put the bacteria solution into $50{ }^{\circ} \mathrm{C}$ water bath for $10 \mathrm{~min}$ and transferred immediately to the ice for 5-10 min. Then the bacteria solution was neutralized with $0.3 \mathrm{~mL}$ of $0.1 \mathrm{M} \mathrm{HCl}$ (for NADH extraction) or $\mathrm{NaOH}$ (for $\mathrm{NAD}^{+}$extraction). After that, the neutralize solution was centrifuged at 12,000 rpm for $5 \mathrm{~min}$, and the supernatants were used for the determination of $\mathrm{NAD}^{+}$ and NADH (San et al. 2002).

\section{Batch fermentation}

Clostridium acetobutylicum was inoculated in liquid RCM for 12-24 h, then transferred to fresh RCM (inoculum size was $5 \% \mathrm{v} / \mathrm{v}$ ). The strain was used as seed when it grown to the logarithmic phase. To study the effect of aspartate on ABE fermentation of $C$. acetobutylicum ATCC 824, different concentrations (0, 1.0, 1.5, 2.0 and $2.5 \mathrm{~g} / \mathrm{L}$ ) of aspartate was added in P2 medium. For fermentation performance tests of the engineered strains, the seed was inoculated in P2 medium. The samples were taken regularly and used for determination of the cell density and the concentration of glucose and products.

\section{Analytical methods}

Cell density was measured by UV spectrophotometer (UV2100, Unico, USA). RT-PCR was performed using fluorescent quantitative PCR (Roche, Switzerland).
The sugar concentration was measured by high performance liquid chromatography (HPLC; Waters 2695, Milford, MA), and the products concentration was measured by gas chromatograph (GC; Agilent 7890A, Agilent Technologies), according to our previous study (Liao et al. 2017).

\section{Results \\ Effect of aspartate on ABE fermentation of C. acetobutylicum ATCC 824}

In order to study the effect of aspartate on $A B E$ fermentation, batch fermentation was carried out by exogenously adding different concentrations of aspartate in P2 medium. As shown in Table 3, the max $\mathrm{OD}_{600}$, sugar consumption and butanol production first increased then decreased slightly with increasing the concentration of aspartate, and the best fermentation performance was obtained when the concentration of aspartate reached $2.0 \mathrm{~g} / \mathrm{L}$. Compared with the control, the max $\mathrm{OD}_{600}$, sugar consumption and butanol production were increased by $17.7 \pm 0.4 \%, 12.5 \pm 1.5 \%$ and $20.7 \pm 2.0 \%$, respectively. In addition, the organic acids concentration was significantly decreased by $42-43.9 \%$ (Table 3). It should be noted that the ethanol production was $\sim 12 \mathrm{~h}$ earlier than that of the control (without aspartate) (Fig. 2).

Table 3 Effect of aspartate on ABE fermentation performance of C. acetobutylicum ATCC 824

\begin{tabular}{|c|c|c|c|c|c|c|c|c|}
\hline $\begin{array}{l}\text { Aspartate } \\
\text { (g/L) }\end{array}$ & Acetone (g/L) & Ethanol (g/L) & Butanol (g/L) & Acetic acid (g/L) & $\begin{array}{l}\text { Butyric acid } \\
(\mathrm{g} / \mathrm{L})\end{array}$ & $\begin{array}{l}\text { Butanol yield } \\
\text { (g/g) }\end{array}$ & $\operatorname{Max} \mathrm{OD}_{600}$ & $\begin{array}{l}\text { Glucose } \\
\text { consumption } \\
\text { (g/L) }\end{array}$ \\
\hline 0 & $5.90 \pm 0.14$ & $0.80 \pm 0.13$ & $11.18 \pm 0.21$ & $1.54 \pm 0.04$ & $1.0 \pm 0.34$ & $0.172 \pm 0.002$ & $6.9 \pm 0.43$ & $64.86 \pm 0.26$ \\
\hline 1.0 & $5.61 \pm 0.55$ & $1.85 \pm 0.10$ & $12.55 \pm 0.21$ & $1.31 \pm 0.04$ & $1.21 \pm 0.24$ & $0.180 \pm 0.004$ & $7.3 \pm 0.32$ & $69.75 \pm 0.49$ \\
\hline 1.5 & $6.18 \pm 0.25$ & $2.06 \pm 0.33$ & $13.24 \pm 0.15$ & $1.21 \pm 0.13$ & $0.51 \pm 0.05$ & $0.187 \pm 0.004$ & $7.7 \pm 0.21$ & $70.79 \pm 0.52$ \\
\hline 2.0 & $6.26 \pm 0.20$ & $1.94 \pm 0.12$ & $13.50 \pm 0.04$ & $1.07 \pm 0.01$ & $0.58 \pm 0.01$ & $0.185 \pm 0.004$ & $8.1 \pm 0.24$ & $72.95 \pm 1.27$ \\
\hline 2.5 & $6.08 \pm 0.17$ & $1.53 \pm 0.08$ & $13.28 \pm 0.10$ & $1.40 \pm 0.06$ & $0.91 \pm 0.06$ & $0.188 \pm 0.002$ & $7.9 \pm 0.14$ & $70.54 \pm 0.33$ \\
\hline
\end{tabular}

\pm , average of three replicates
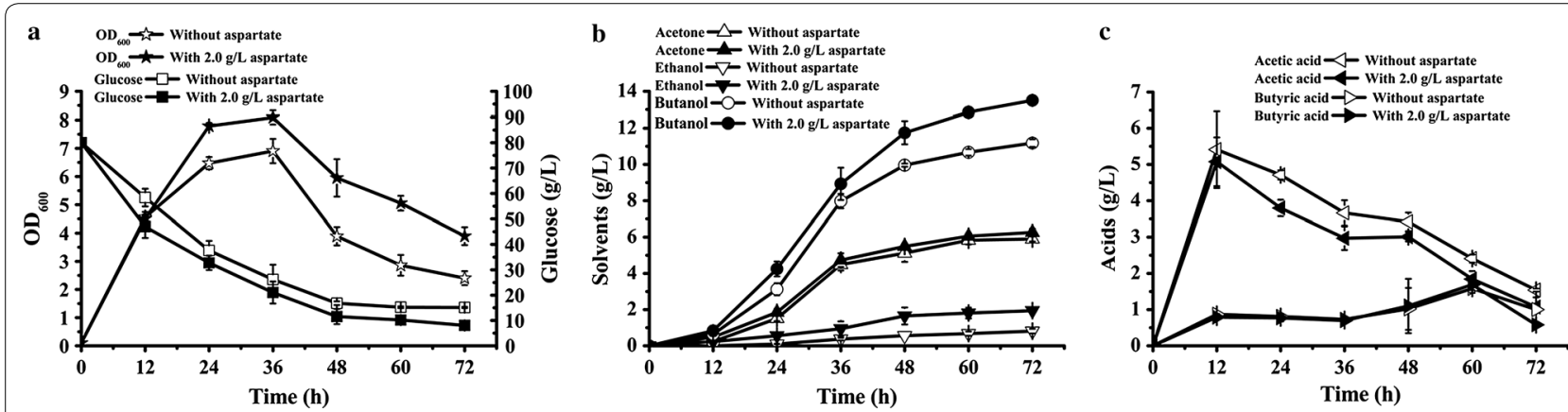

Fig. 2 ABE fermentation with or without aspartate (2.0 g/L). a Growth and glucose consumption; b solvents production; corganic acids production 
Effect of aspartate on the transcription of NADH de novo synthesis genes and the intracellular $N A D(H)$ biosynthesis of $C$. acetobutylicum ATCC 824

Based on the genomic sequence analysis, we speculated that the de novo synthesis of NADH in C. acetobutylicum ATCC 824 is through Asp pathway, which using aspartate as the precursor. To investigate the effect of aspartate on $\mathrm{NAD}(\mathrm{H})$ synthesis, the transcription of NADH de novo synthesis genes ( $\operatorname{nadA}, \operatorname{nadB}$ and $\operatorname{nad} C$ ) and the concentration of intracellular $\mathrm{NAD}(\mathrm{H})$ were detected when $2.0 \mathrm{~g} / \mathrm{L}$ aspartate was added (no aspartate addition was used as control). As shown in Fig. 3, in general, the transcription level of $n a d A, n a d B$ and $n a d C$ increased first and then decreased from 12 to $36 \mathrm{~h}$. When $2.0 \mathrm{~g} / \mathrm{L}$ aspartate was added, the transcription levels of all these three genes were consistently higher than that of the control. Furthermore, the concentration of intracellular $\mathrm{NAD}^{+}, \mathrm{NADH}$ and total $\mathrm{NAD}(\mathrm{H})$ were increased from $1.46 \pm 0.09,2.92 \pm 0.05$ and $4.38 \pm 0.04 \mu \mathrm{M} / \mathrm{OD}_{600}$ to $2.37 \pm 0.04,5.39 \pm 0.04$ and $7.76 \pm 0.42 \mu \mathrm{M} / \mathrm{OD}_{600}$, respectively (Fig. 4).

\section{Effect of overexpressing the NADH de novo synthesis genes on $A B E$ fermentation}

As exogenously addition of the NADH de novo synthesis precursor (aspartate) could improve the butanol production by promoting the biosynthesis of $\operatorname{NAD}(\mathrm{H})$, then we speculated that the butanol production could be promoted by enhancing the de novo synthesis pathway of NADH. Therefore, the essential genes for the de novo synthesis of NADH ( $\operatorname{lad} A$, nadB and/or $n a d C$ ) has been overexpressed in C. acetobutylicum ATCC 824, and only three engineered strains $824(\operatorname{nad} A), 824(\operatorname{nadB})$ and $824($ nadC) were finally obtained. As shown in Table 4 , the butanol titer of engineered strains 824(nadA), 824(nadB) and $824(\operatorname{nadC})$ was $11.42 \pm 0.13 \mathrm{~g} / \mathrm{L}, 11.39 \pm 0.21 \mathrm{~g} / \mathrm{L}$ and

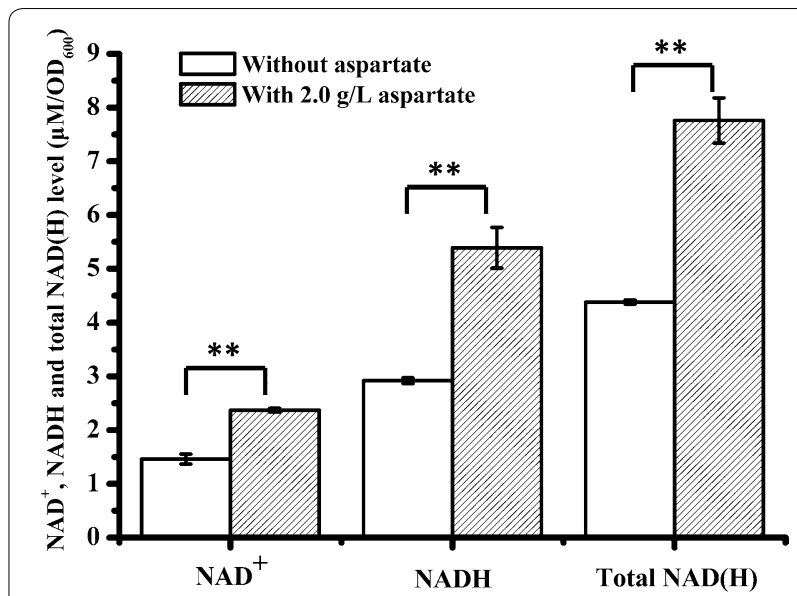

Fig. $4 \mathrm{NAD}^{+}, \mathrm{NADH}$ and total $\mathrm{NAD}(\mathrm{H})$ concentration of $\mathrm{C}$. acetobutylicum ATCC 824 with or without aspartate $(2.0 \mathrm{~g} / \mathrm{L})$. The data are the means and standard deviations of three replicates $(* * \mathrm{P} \leq 0.01$; t-test)

$11.82 \pm 0.11 \mathrm{~g} / \mathrm{L}$, respectively, which were higher than that of the wild type strain $(10.41 \pm 0.06 \mathrm{~g} / \mathrm{L})$.

\section{Effect of enhancing the VB1 biosynthesis pathway and NADH de novo synthesis pathway simultaneously on $A B E$ fermentation}

To further improve the butanol production, VB1 biosynthesis related genes thiC, thiG and thiE and NADH de novo synthesis gene nadC were co-expressed in $C$. acetobutylicum ATCC 824. The results showed that the butanol titer of engineered strain 824(thiCGEnadC) reached $13.96 \pm 0.11 \mathrm{~g} / \mathrm{L}$, which was significantly higher than that of engineered strains 824(thiCGE) $(13.02 \pm 0.15 \mathrm{~g} / \mathrm{L})$ and $824(\operatorname{nadC})(11.82 \pm 0.11 \mathrm{~g} / \mathrm{L})$, as well as the wild type strain $(10.41 \pm 0.06 \mathrm{~g} / \mathrm{L})$ (Table 4$)$.
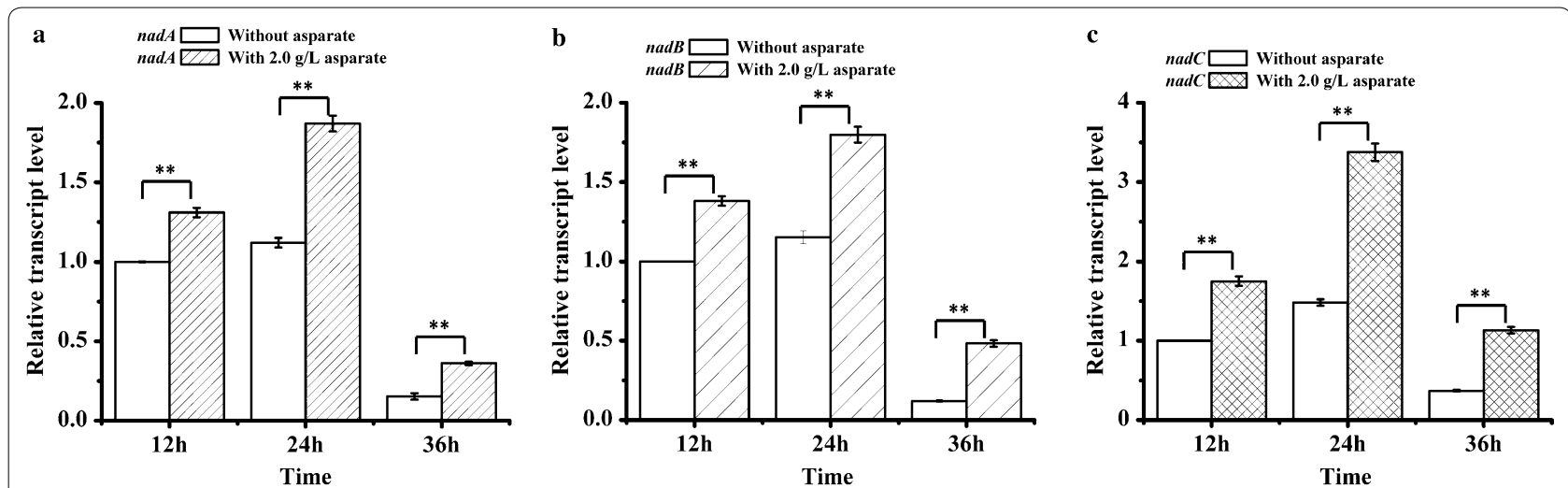

Fig. 3 RT-PCR analysis the transcriptional level of NADH de novo synthesis genes. a The transcriptional level of nadA; $\mathbf{b}$ the transcriptional level of $n a d B ; \mathbf{c}$ the transcriptional level of $n a d C$. The data are the means and standard deviations of three replicates (**P $\leq 0.01 ; t$ test) 
Table 4 Comparison of fermentation performance of C. acetobutylicum ATCC 824 and recombinant strains

\begin{tabular}{llllllll}
\hline Strains & Acetone (g/L) & Ethanol (g/L) & Butanol (g/L) & Acetic acid (g/L) & Butyric acid (g/L) & $\begin{array}{c}\text { Butanol yield (g/g) } \\
\text { Glucose } \\
\text { consumption } \\
\text { (g/L) }\end{array}$ \\
\hline ATCC 824 & $5.78 \pm 0.19$ & $0.44 \pm 0.07$ & $10.41 \pm 0.06$ & $3.83 \pm 0.13$ & $1.34 \pm 0.05$ & $0.167 \pm 0.002$ & $62.34 \pm 1.21$ \\
824(nadA) & $6.21 \pm 0.14$ & $1.05 \pm 0.14$ & $11.42 \pm 0.13$ & $3.23 \pm 0.14$ & $1.16 \pm 0.21$ & $0.170 \pm 0.001$ & $67.18 \pm 0.58$ \\
824(nadB) & $6.11 \pm 0.09$ & $0.76 \pm 0.11$ & $11.39 \pm 0.21$ & $3.36 \pm 0.12$ & $1.25 \pm 0.15$ & $0.171 \pm 0.006$ & $66.61 \pm 1.04$ \\
824(nadC) & $6.15 \pm 0.05$ & $0.85 \pm 0.05$ & $11.82 \pm 0.11$ & $3.05 \pm 0.21$ & $1.11 \pm 0.12$ & $0.176 \pm 0.001$ & $67.16 \pm 0.64$ \\
824(thiCGE) & $6.71 \pm 0.16$ & $1.85 \pm 0.07$ & $13.02 \pm 0.15$ & $1.80 \pm 0.26$ & $0.73 \pm 0.11$ & $0.186 \pm 0.001$ & $69.88 \pm 0.55$ \\
824(thiCGE-nadC) & $6.97 \pm 0.75$ & $1.35 \pm 0.01$ & $13.96 \pm 0.11$ & $2.46 \pm 0.08$ & $0.94 \pm 0.19$ & $0.188 \pm 0.001$ & $74.23 \pm 0.52$ \\
\hline
\end{tabular}

\pm , average of three replicates

\section{Discussions}

The co-factor NADH plays an important role in butanol biosynthesis, and insufficient intracellular NADH supply of solventogenic Clostridium limits the production of butanol ( $\mathrm{Li}$ et al. 2015; Qi et al. 2018). Therefore, increasing the supply of NADH is of great significance for butanol production. Although a lot of work has been done to improve the butanol production by increasing the NADH level of solventogenic Clostridium in recent years (Cooksley et al. 2012; Liu et al. 2016; Qi et al. 2018; Ventura et al. 2013), there were few researches focus on increasing the NADH levels by enhancing the de novo synthesis pathways of NADH. Previous research showed that the NAD(P)H level of Clostridium species strain $\mathrm{BOH} 3$ was increased by $67 \%$ with exogenously tryptophan addition (the precursor of NAD(P)H de novo synthesis) (Li et al. 2015). When cassava hydrolysate was used as substrate for ABE fermentation, butanol titer was $68 \%$ higher than that of no tryptophan addition. This means that it could be an effective way to increase the NADH level by enhancing the de novo synthesis pathways of NADH. For de novo synthesis pathways of NADH, aspartate is the precursor of Asp pathway, while tryptophan is the precursor of kynurenine pathway (Akira et al. 2006) (Fig. 1). Based on the genome sequencing data of $C$. acetobutylicum ATCC 824 (Nölling et al. 2001), we found that it could use aspartate as the precursor for NADH de novo synthesis, rather than tryptophan. Therefore, the effect of aspartate on $\mathrm{ABE}$ fermentation of $C$. acetobutylicum ATCC 824 has been studied. As expected, with the increasing of aspartate concentration, the fermentation performance of $C$. acetobutylicum ATCC 824 was significantly enhanced. It was showed the optimum concentration of aspartate was $2.0 \mathrm{~g} / \mathrm{L}$, and the max $\mathrm{OD}_{600}$, sugar consumption and butanol production reached $8.1 \pm 0.24, \quad 71.95 \pm 1.27 \mathrm{~g} / \mathrm{L}$ and $13.50 \pm 0.04 \mathrm{~g} / \mathrm{L}$, improved by $17.7 \pm 0.4 \%, 12.5 \pm 1.5 \%$ and $20.7 \pm 2.0 \%$ compared with those of the control, respectively (Fig. 2 and Table 3). These results indicated that aspartate could promote $\mathrm{ABE}$ fermentation of $C$. acetobutylicum ATCC 824. The effect of tryptophan on ABE fermentation has also been studied, however, the results showed that tryptophan had an inhibitory effect on ABE fermentation (Additional file 1: Table S1).

Although we speculated that aspartate is the precursor of NADH de novo synthesis in C. acetobutylicum ATCC 824, it has never been proved. In order to investigate the relationship between aspartate and $\mathrm{NAD}(\mathrm{H})$, the effect of aspartate on $\mathrm{NAD}(\mathrm{H})$ biosynthesis has been studied. Firstly, the expression of NADH de novo synthesis genes has been analyzed by RT-PCR. As a result, when $2.0 \mathrm{~g} / \mathrm{L}$ aspartate was added, the transcription level of nadA, nadB and nadC in C. acetobutylicum ATCC 824 were significantly higher than that of without aspartate addition (Fig. 3). In addition, the $\operatorname{NAD}(\mathrm{H})$ concentration has also been detected. The result showed that intracellular $\mathrm{NAD}^{+}, \mathrm{NADH}$ and total $\mathrm{NAD}(\mathrm{H})$ of $C$. acetobutylicum ATCC 824 were significantly increased when $2.0 \mathrm{~g} / \mathrm{L}$ aspartate was added, which were $63.9 \pm 8.0 \%, 85.0 \pm 16.5 \%$ and $77.7 \pm 8.0 \%$ higher than those of the control (no aspartate addition), and the $\mathrm{NADH} / \mathrm{NAD}^{+}$ratio was improved by $12.7 \pm 2.9 \%$ (Fig. 4). These results indicated that aspartate could promote the biosynthesis of $\operatorname{NAD}(\mathrm{H})$. Therefore, the increasement of NADH concentration resulted in a reduced organic acids production, increased butanol and ethanol production, and an early production of ethanol (Table 3 and Fig. 2). For example, compared to the control, when $2.0 \mathrm{~g} / \mathrm{L}$ aspartate was added, the butanol/acetone ratio was increased from $1.89 \pm 0.01$ to $2.11 \pm 0.01$, and the butanol/organic acids ratio was increased from $4.47 \pm 0.62$ to $8.18 \pm 0.16$. These results suggested that the carbon metabolism was directed to the reduced product (butanol and ethanol) synthesis under sufficient NADH supply, which were consistent with previous studies (Hönicke et al. 2012; Li et al. 2015). When $1 \mathrm{mM} \mathrm{MV} \mathrm{(an} \mathrm{artificial}$ 
electron carrier which can inhibit the hydrogenase activity) was added in the medium to increase the availability of the intracellular $\mathrm{NAD}(\mathrm{P}) \mathrm{H}$ of $C$. acetobutylicum ATCC 824, the butanol titer was improved by $23 \%$, ethanol titer was improved by $40 \%$, resulting in a higher butanol/acetone ratio (12.4 vs. 2.3) as compared to the control (Hönicke et al. 2012). Similarly, when $5 \mathrm{mM}$ tryptophan (the precursor of $\mathrm{NAD}(\mathrm{P}) \mathrm{H}$ de novo synthesis) was added to improve the NAD(P)H levels of Clostridium sp. strain $\mathrm{BOH} 3$, the butanol titer was significantly improved and organic acids (acetic acid and butyric acid) titers were significantly reduced, which resulted in higher butanol/acetone ratio (6.6 vs. 3.7) and butanol/bioacid ratio (2.1 vs. 1.1) as compared to the control (Li et al. 2015). All of these researches indicated that the increasement of NADH had an remarkable effect on the metabolic distribution, and could promote the biosynthesis of butanol or other reducing products (BerríOs-Rivera et al. 2002; Jing et al. 2015; Li et al. 2015; Qi et al. 2018; Saini et al. 2016).

Previous studies have shown that $\operatorname{nad} A, \operatorname{nadB}$ and nadC were the essential structural genes of the NADH de novo synthesis pathway (Haruhiko et al. 2010; Sun and Setlow 1993; Zhou et al. 2011). In addition, the expression of $n a d A, n a d B$ and $n a d C$ were significantly up-regulated in $C$. acetobutylicum ATCC 824 after aspartate addition (Fig. 3). Therefore, the effect of overexpression of $\operatorname{nadA}$, $\operatorname{nadB}$ and $\operatorname{nadC}$ on ABE fermentation has been studied in this study. As expected, the butanol production was improved by overexpressing these genes (Table 4). And the engineered strain 824(nadC) showed the best fermentation performance. For $824(\operatorname{nadC})$, the butanol titer was $13.5 \pm 0.4 \%$ higher than that of the wild type strain, and the organic acids titer was decreased obviously (Table 4). These results indicated that with the enhancement of NADH de novo synthesis pathway, the carbon metabolism was directed to butanol synthesis. It is noteworthy that the fermentation performance of the recombinant strains overexpressing the essential genes of the de novo synthesis of NADH was inferior to that of exogenously aspartate addition, which can be attributed to the following reasons: on the one hand, single gene overexpression could not effectively strengthen the NADH de novo synthesis pathway (we have tried to co-express all of the essential genes of NADH de novo synthesis pathway, however, the recombinant vector was not successfully constructed despite a great deal of effort); on the other hand, the effect of aspartate on ABE fermentation may be attributed not only to the increasement of intracellular NADH level, but also to other factors, such as aspartate could be used as carbon and nitrogen nutrient sources or could be converted into some intermediates which were essential to cell physiology and biochemistry (Fernández and Zúñiga 2006). The beneficial effect of aspartate on $\mathrm{ABE}$ fermentation by $C$. acetobutylicum ATCC 824 need to be further explored.

Previous studies showed that butanol production could be significantly improved by increasing intracellular ATP and NADH levels simultaneously (Liu et al. 2016; Ventura et al. 2013). For example, the intracellular ATP and NADH levels were simultaneously increased by overexpressing of pyruvate kinase $(p y k A)$ gene and 6-phosphofructose kinase gene ( $p f k A)$, and this eventually led to a $29.4 \%$ increase in butanol production of $C$. acetobutylicum ATCC 824 (Ventura et al. 2013). The ATP and NADH levels of $C$. beijerinckii were also increased by knocking out the gene Cbei_4110, encoding the $\mathrm{NADH}$-quinone oxidoreductase, which is a membranebound enzyme and associated with energy metabolism and electron transport, and resulted in a $21.8 \%$ increase in butanol titer (Liu et al. 2016). Our previous study showed that the sugar consumption and ATP production of C. acetobutylicum ATCC 824 were improved by overexpressing the VB1 biosynthesis related genes thiC, thiG and thiE (Liao et al. 2018), as a result, the butanol production was significantly increased. Therefore, to further improve the butanol production, VB1 biosynthesis related genes thiC, thiG and thiE and NADH de novo synthesis gene $n a d C$ were co-expressed in C. acetobutylicum ATCC 824 to promote the biosynthesis of NADH and ATP simultaneously. The results showed that the butanol titer of 824(thiCGE-nadC) reached $13.96 \pm 0.11 \mathrm{~g} / \mathrm{L}$, which were $7.2 \pm 0.4 \%, 18.1 \pm 0.1 \%$ and $34.1 \pm 0.1 \%$ higher than that of 824 (thiCGE), 824(nadC) and the wild type strain, respectively (Table 4). This result indicated that the butanol production could be further improved by simultaneously increasing the levels of ATP and NADH.

In conclusion, we found that aspartate could be used as the precursor of NADH de novo synthesis to promote the $\mathrm{NAD}(\mathrm{H})$ biosynthesis in $C$. acetobutylicum ATCC 824, then resulted in an improvement in butanol production. Furthermore, the butanol production could be improved by overexpressing the NADH de novo synthesis genes. And the butanol titer could be further increased by strengthening the VB1 biosynthesis and NADH de novo synthesis pathway simultaneously. This study has a reference value for the NADH related researches of other microbes, and the engineering strategy used in this study provides a new idea for construction of efficient fuel-producing strains.

\section{Supplementary information}

Supplementary information accompanies this paper at https://doi. org/10.1186/s13568-019-0874-6. 
Additional file 1: Table S1. Effect of tryptophan on ABE fermentation performance of C. acetobutylicum ATCC 824.

\section{Abbreviations}

ABE: acetone-butanol-ethanol; LB: Luria-Bertani; RCM: reinforced clostridial medium; HPLC: high performance liquid chromatography; GC: gas chromatograph.

\section{Acknowledgements}

We thank Professor Shang-Tian Yang (The Ohio State University) for providing the strain C. acetobutylicum ATCC 824.

\section{Authors' contributions}

$Z L$ and $X Y$ carried out experiments and $Z L$ drafted the manuscript. JW and HF are responsible for this study, participated its design. JW and HF discussed and suggested with the manuscript. All authors read and approved the final manuscript.

\section{Funding}

This work was supported by the National Natural Science Foundation of China (21808069, 21878103 and 21676098), the Natural Science Foundation of Guangdong Province of China (2018A030310368), the State Key Laboratory of Pulp and Paper Engineering (2017C03), the China Postdoctoral Science Foundation (2017M612667, 2018T110871), and the Fundamental Research Funds for the Central Universities (2017BQ084, 2017PY013).

\section{Availability of data and materials}

The datasets supporting the conclusions of this article are included within the article.

\section{Ethics approval and consent to participate}

Not applicable as this article does not contain any studies with human participants or animals performed by any of the authors.

\section{Consent for publication}

Not applicable.

\section{Competing interests}

The authors declare that they have no competing interests.

\section{Author details}

'School of Biology and Biological Engineering, South China University of Technology, Guangzhou 510006, China. ${ }^{2}$ State Key Laboratory of Pulp and Paper Engineering, South China University of Technology, Guangzhou 510640, China.

Received: 12 August 2019 Accepted: 5 September 2019

Published online: 10 September 2019

\section{References}

Akira K, Kazuya U, Mitsuru A, Takashi H (2006) Early steps in the biosynthesis of NAD in Arabidopsis start with aspartate and occur in the plastid. Plant Physiol 141(3):851-857

Balzer GJ, Thakker C, Bennett GN, San KY (2013) Metabolic engineering of Escherichia coli to minimize byproduct formate and improving succinate productivity through increasing NADH availability by heterologous expression of $\mathrm{NAD}^{+}$-dependent formate dehydrogenase. Metab Eng 20(5):1-8

BerríOs-Rivera SJ, Bennett GN, San KY (2002) The effect of increasing NADH availability on the redistribution of metabolic fluxes in Escherichia coli chemostat cultures. Metab Eng 4(3):230-237

Biswas R, Zheng T, Olson DG, Lynd LR, Guss AM (2015) Elimination of hydrogenase active site assembly blocks $\mathrm{H} 2$ production and increases ethanol yield in Clostridium thermocellum. Biotechnol Biofuels 8(1):20

Cooksley CM, Zhang Y, Wang H, Redl S, Winzer K, Minton NP (2012) Targeted mutagenesis of the Clostridium acetobutylicum acetone-butanol-ethanol fermentation pathway. Metab Eng 14(6):630-641
Fernández M, Zúñiga M (2006) Amino acid catabolic pathways of lactic acid bacteria. Crit Rev Microbiol 32(3):155-183

Haruhiko T, Masako S, Masayuki I, Hideaki Y (2010) Regulation of the expression of genes involved in NAD de novo biosynthesis in Corynebacterium glutamicum. Appl Environ Microbiol 76(16):5488-5495

Heap JT, Pennington OJ, Cartman ST, Carter GP, Minton NP (2007) The ClosTron: a universal gene knock-out system for the genus Clostridium. J Microbiol Methods 70(3):452-464

Heap JT, Pennington OJ, Cartman ST, Minton NP (2009) A modular system for Clostridium shuttle plasmids. J Microbiol Methods 78(1):79-85

Hönicke D, Janssen H, Grimmler C, Ehrenreich A, Lütke-Eversloh T (2012) Global transcriptional changes of Clostridium acetobutylicum cultures with increased butanol:acetone ratios. New Biotechnol 29(4):485-493

Jang YS, Im JA, Choi SY, Lee Jl, Sang YL (2014) Metabolic engineering of Clostridium acetobutylicum for butyric acid production with high butyric acid selectivity. Metab Eng 23(2):165-174

Jing F, Zhiwen W, Tao C, Weixi L, Ting S, Guanglu W, Ya-Jie T, Xueming Z (2015) $\mathrm{NADH}$ plays the vital role for chiral pure $\mathrm{D}-(-)-2,3$-butanediol production in Bacillus subtilis under limited oxygen conditions. Biotechnol Bioeng 111(10):2126-2131

Kurnasov O, Goral V, Colabroy K, Gerdes S, Anantha S, Osterman A, Begley TP (2003) NAD biosynthesis: identification of the tryptophan to quinolinate pathway in bacteria. Chem Biol 10(12):1195-1204

Lee SY, Park JH, Jang SH, Nielsen LK, Kim J, Jung KS (2008) Fermentative butanol production by clostridia. Biotechnol Bioeng 101(2):209-228

Li T, Yan Y, He J (2015) Enhanced direct fermentation of cassava to butanol by Clostridium species strain $\mathrm{BOH} 3$ in cofactor-mediated medium. Biotechnol Biofuels 8(1):166

Liao Z, Zhang Y, Luo S, Suo Y, Zhang S, Wang J (2017) Improving cellular robustness and butanol titers of Clostridium acetobutylicum ATCC824 by introducing heat shock proteins from an extremophilic bacterium. J Biotechnol 252:1-10

Liao Z, Suo Y, Xue C, Fu H, Wang J (2018) Improving the fermentation performance of Clostridium acetobutylicum ATCC 824 by strengthening the VB1 biosynthesis pathway. Appl Microbiol Biot 102(18):8107-8119

Lim JH, Sang WS, Kim SY, Jung GY (2013) Model-driven rebalancing of the intracellular redox state for optimization of a heterologous $n$-butanol pathway in Escherichia coli. Metab Eng 20(5):56-62

Liu D, Chen Y, Ding F, Guo T, Xie J, Zhuang W, Niu H, Shi X, Zhu C, Ying H (2015) Simultaneous production of butanol and acetoin by metabolically engineered Clostridium acetobutylicum. Metab Eng 27:107-114

Liu J, Guo T, Wang D, Shen X, Liu D, Niu H, Liang L, Ying H (2016) Enhanced butanol production by increasing NADH and ATP levels in Clostridium beijerinckii NCIMB 8052 by insertional inactivation of Cbei_4110. Appl Microbiol Biot 100(11):4985-4996

Nielsen J (2003) It is all about metabolic fluxes. J Bacteriol 185(24):7031-7035

Nölling J, Breton G, Omelchenko MV, Makarova KS, Zeng Q, Gibson R, Lee HM, Dubois J, Qiu D, Hitti J, Sequencing Center Production Finishing GTC, Teams B, Wolf YI, Tatusov RL, Sabathe F, Doucette-Stamm L, Soucaille P, Daly MJ, Bennett GN, Koonin EV, Smith DR (2001) Genome sequence and comparative analysis of the solvent-producing bacterium Clostridium acetobutylicum. J Bacteriol 183(16):4823-4838

Peguin S, Goma G, Delorme P, Soucaille P (1994) Metabolic flexibility of Clostridium acetobutylicum in response to methyl viologen addition. Appl Microbiol Biot 42(4):611-616

Qi F, Thakker C, Zhu F, Pena M, San K-Y, Bennett GN (2018) Improvement of butanol production in Clostridium acetobutylicum through enhancement of NAD(P)H availability. J Ind Microbiol Biot 45(11):993-1002

Saini M, Li S-Y, Wang ZW, Chiang C-J, Chao Y-P (2016) Systematic engineering of the central metabolism in Escherichia coli for effective production of n-butanol. Biotechnol Biofuels 9(1):69

San KY, Bennett GN, Berríos-Rivera SJ, Vadali RV, Yang YT, Horton E, Rudolph FB, Sariyar B, Blackwood K (2002) Metabolic engineering through cofactor manipulation and its effects on metabolic flux redistribution in Escherichia coli. Metab Eng 4(2):182-192

Shen CR, Lan El, Yasumasa D, Antonino B, Kwang Myung C, Liao JC (2011) Driving forces enable high-titer anaerobic 1-butanol synthesis in Escherichia coli. Appl Environ Microbiol 77(9):2905-2915

Sun D, Setlow P (1993) Cloning, nucleotide sequence, and regulation of the Bacillus subtilis nadB gene and a nifS-like gene, both of which are essential for NAD biosynthesis. J Bacteriol 175(5):1423-1432 
Tomas CA, Welker NE, Papoutsakis ET (2003) Overexpression of groESL in Clostridium acetobutylicum results in increased solvent production and tolerance, prolonged metabolism, and changes in the cell's transcriptional program. Appl Environ Microbiol 69(8):4951-4965

Tseng GC, Oh M-K, Rohlin L, Liao JC, Wong WH (2001) Issues in cDNA microarray analysis: quality filtering, channel normalization, models of variation and assessment of gene effects. Nucleic Acids Res 29(12):2549-2557

Ventura J-RS, Hu H, Jahng D (2013) Enhanced butanol production in Clostridium acetobutylicum ATCC 824 by double overexpression of 6-phosphofructokinase and pyruvate kinase genes. Appl Microbiol Biot 97(16):7505-7516

Wang S, Zhang Y, Dong H, Mao S, Zhu Y, Wang R, Luan G, Li Y (2011) Formic acid triggers the "Acid Crash" of acetone-butanol-ethanol fermentation by Clostridium acetobutylicum. Appl Environ Microbiol 77(5):1674-1680
Wietzke M, Bahl H (2012) The redox-sensing protein Rex, a transcriptional regulator of solventogenesis in Clostridium acetobutylicum. Appl Microbiol Biot 96(3):749-761

Zhou Y, Wang L, Yang F, Lin X, Zhang S, Zhao ZK (2011) Determining the extremes of the cellular NAD(H) level by using an Escherichia coli $\mathrm{NAD}^{+}$-auxotrophic mutant. Appl Environ Microbiol 77(17):6133

\section{Publisher's Note}

Springer Nature remains neutral with regard to jurisdictional claims in published maps and institutional affiliations.

\section{Submit your manuscript to a SpringerOpen ${ }^{\circ}$ journal and benefit from:}

- Convenient online submission

- Rigorous peer review

- Open access: articles freely available online

- High visibility within the field

- Retaining the copyright to your article

Submit your next manuscript at $\boldsymbol{\nabla}$ springeropen.com 